\title{
Iterative Linearization Methods suited for Digital Pre-distortion of Power Amplifiers
}

\author{
Ernst Aschbacher, Mathias Steinmair, Markus Rupp \\ Institute of Communications and Radio-Frequency Engineering \\ University of Technology Vienna \\ Gusshausstrasse 25/389, A-1040 Vienna, Austria \\ Email: \{easchb,mrupp $\} @$ tuwien.ac.at \\ Web: http://www.nt.tuwien.ac.at/rapid_prototyping
}

\begin{abstract}
This paper deals with iterative linearization of a nonlinear dynamical system. The methods proposed are suited for digital pre-distortion of power amplifiers working close to saturation, as it is common for efficiency reasons. Two methods (a well known successive approximation method based on a fixed-point formulation and a Newton method) are compared with respect to convergence speed, robustness against model uncertainties, and complexity. The presented methods are used to linearize a Volterra model of a measured power amplifier.
\end{abstract}

\section{INTRODUCTION}

Digital pre-distortion is a promising technique to linearize the signal path with a power amplifier working near saturation. Highly linear and at the same time highly efficient amplifiers are not available. Digital pre-distortion works entirely in the digital domain and is already in use in $2 \mathrm{G}$ systems. The techniques are mostly based on look-up tables (LUTs) [1], using a memoryless characteristic of the amplifier. With large signal bandwidths as in $3 \mathrm{G}$ systems, memory effects become more pronounced [2]. The LUTs for such systems are not manageable and other techniques are to be found. Typical wide-band models for power amplifiers are Volterra series and specializations like Wiener- and Hammerstein-systems [3]. Analytic inverses of these systems are often not known. For Volterra models an inverse up to some order $p$ is known [4], but rather complex.

In this contribution attention is focused on techniques which solve the linearization problem in an iterative and approximate way. Two methods, one based on successive approximation, investigated in [5], and one based on a Newton method are compared.

\section{ITERATIVE LINEARIZATION}

The problem is to find a structure that is able to linearize a given nonlinear system if placed in front of it. This can be solved in an iterative way, using a known successive approximation method [5], based on a fixed-point equation, and a Newton method, developed in this contribution.

Research reported here was performed in the context of the network TARGET (Top Amplifier Research Groups in a European Team) and supported by the Information Society Technologies Programme of the EU under contract IST-1-507893-NOE, www.target-org.net
Given a model $\mathbb{N}$ of a nonlinear system, a structure $\mathbb{P}$ is searched which, placed before the nonlinear system, linearizes the signal path as good as possible. Mathematically

$$
\mathbb{N}(\mathbb{P}(u[n]))=\mathbb{L}(u[n]),
$$

whereby $u[n]$ denotes the input sequence and $\mathbb{L}$ is a linear operator, see Fig. 1. Searching the unknown pre-inverse $\mathbb{P}$ is equivalent to determining the signal $\mathbb{P}(u[n])=y[n]$ - given both signals $u[n]$ and $y[n]$, the pre-inverse is also known.

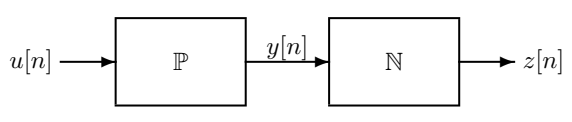

Fig. 1. Pre-distortion problem

Since the system is not exactly known, modeling errors, consisting of structural mismatches between the real system and the model and model-parameter estimation errors will ultimately limit the achieved linearity, see Section III further ahead.

\section{A. Successive approximation}

Setting $\mathbb{P}(u[n])=y[n]$, assuming that the nonlinear model $\mathbb{N}$ can be decomposed in an additive way

$$
\mathbb{N}(y[n])=\sum_{p=1}^{P} \mathbb{N}_{p}(y[n]),
$$

(1) can be rewritten as

$$
\mathbb{L}(u[n])=\mathbb{N}_{1}(y[n])+\sum_{p=2}^{P} \mathbb{N}_{p}(y[n]) .
$$

Assuming that $\mathbb{N}_{1}$ is invertible, this can be turned in

$$
y[n]=\mathbb{N}_{1}^{-1}\left(\mathbb{L}(u[n])-\sum_{p=2}^{P} \mathbb{N}_{p}(y[n])\right) .
$$

Equation (4) denotes a fixed-point equation in $y[n]$,

$$
y[n]=\mathbb{T}(y[n]),
$$


which can be solved using a successive approximation approach,

$$
y_{i+1}[n]=\mathbb{T}\left(y_{i}[n]\right), y_{0}[n] \text { given } .
$$

The method converges if $\mathbb{T}(\cdot)$ is a contraction mapping for the signals involved [5]. The complexity (e.g. number of multiplications) of this method grows linearly with the numbers of iterations. The convergence rate is also of linear order, meaning that for high accuracy a large number of iterations is necessary.

\section{B. Newton method}

Alternatively, (1) can be rewritten as

$$
\mathbb{N}(y[n])-\mathbb{L}(u[n])=z[n]-\mathbb{L}(u[n])=\mathbb{S}(y[n])=0 .
$$

The root of this equation corresponds to the signal after the pre-inverse. It can iteratively be found with a Newton procedure [6]

$$
y_{i+1}[n]=y_{i}[n]-\left(\mathbb{S}^{\prime}\left(y_{i}[n]\right)\right)^{-1} \mathbb{S}\left(y_{i}[n]\right), y_{0}[n] \text { given. }
$$

Newtons method can be seen as a successive approximation with

$$
y_{i+1}[n]=\mathbb{T}\left(y_{i}[n]\right)=y_{i}[n]-\left(\mathbb{S}^{\prime}\left(y_{i}[n]\right)\right)^{-1} \mathbb{S}\left(y_{i}[n]\right) .
$$

For a proof of convergence, an initial approach would be to determine if $\left\|\mathbb{T}^{\prime}(y[n])\right\|<1$, meaning that $\mathbb{T}$ is a contraction mapping [6].

In contrast to the fixed-point method, the model $\mathbb{N}$ must not be decomposable additively - this enlarges the class of models which can be linearized. Further, this method does not require the inversion of the operator $\mathbb{N}_{1}$ in (4). If $\mathbb{N}$ is a Volterra filter, $\mathbb{N}_{1}$ is a linear filter, which could not be stably inverted. A restriction of this method is that the operator $\mathbb{S}$ must be analytic, otherwise the derivative cannot be computed. The convergence rate of this method is of quadratic order, in contrast to the linear convergence of the successive approximation. These advantages have to be paid with more complexity, especially the inversion of the matrix $\mathbb{S}^{\prime}$ is costly.

The derivative $\mathbb{S}^{\prime}$ is

$$
\mathbb{S}^{\prime}=\left(\begin{array}{ccccc}
\frac{\partial z[n]}{\partial y[n]} & \frac{\partial z[n]}{\partial y[n-1]} & \frac{\partial z[n]}{\partial y[n-2]} & \ldots & \ldots \\
0 & \frac{\partial z[n-1]}{\partial y[n-1]} & \frac{\partial z[n-1]}{\partial y[n-2]} & \frac{\partial z[n-1]}{\partial y[n-3]} & \ldots \\
\vdots & \vdots & \vdots & \vdots & \ddots
\end{array}\right)
$$

and does not depend on $\mathbb{L}$. Since $\mathbb{N}$ is assumed to be causal, $\mathbb{S}^{\prime}$ is upper triangular. If the memory of $\mathbb{N}$ is finite, only a band in the upper part of width equal to the memory length is not equal to zero. Assuming further an observation interval of $L$, this becomes an $L \times L$ matrix. This matrix has to be evaluated at $y_{i}[n]$ and inverted, which yields an upper triangular matrix.

\section{Approximate Newton iteration}

A first heuristic and very rough approximation of $\mathbb{S}^{\prime}$ in order to reduce complexity consists of neglecting the off-diagonal elements. The equation (8) simplifies with this to

$$
y_{i+1}[n]=y_{i}[n]-\left(\left.\frac{\partial \mathbb{N}}{\partial y[n]}\right|_{y_{i}[n]}\right)^{-1} \mathbb{S}\left(y_{i}[n]\right), y_{0}[n] \text { given. }
$$

The derivative $\mathbb{S}^{\prime}$ in (10) reduces from a matrix to a scalar - the inversion now being a simple division. This scalar is the outcome of an operator of nonlinear order one less than $\mathbb{N}$. It has to be evaluated at $y_{i}[n]$. Since it is in general an operator with memory, passed elements of the sequence $y_{i}[n]$ will influence the outcome. Since at time $n$ the solution of the Newton iteration $y_{I}[n-m], m>0, I$ denoting the total number of iterations, is already known, these values can replace passed values in the sequence $y_{i}[n]$, specifically $\mathbb{N}^{\prime}\left(y_{i}[n], y_{I}[n-1], y_{I}[n-2], \ldots\right)$, see Fig. 2 for a schematic representation. Assuming that $y_{I}[n-m]$ is closer to the root $y[n-m]$ for $m>0$, this is expected to improve the convergence speed.

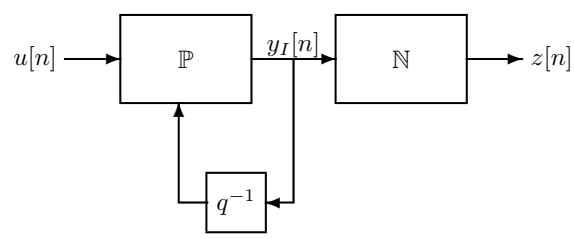

Fig. 2. Pre-distortion with feedback of the output signal

If (8) is not seen in terms of operators which map signals into signals but in terms of functionals which map a signal into only one sample, it can be easily seen that the proposed algorithm is an exact Newton method on a sample-by-sample basis.

\section{Complexity}

The complexity of the fixed-point method consists of evaluating $I$ times the operation $\mathbb{T}$ if $I$ is the total number of iterations performed. If $\mathbb{N}$ is a Volterra system of nonlinear order $P$ and memory length $N$, equal for each kernel, this gives in total (for real valued signals)

$$
M_{F x p}=I \sum_{p=1}^{P} p\left(\begin{array}{c}
p+N-1 \\
p
\end{array}\right)=I M_{P, N}
$$

multiplications. The evaluation of $\mathbb{L}(u[n])$ is neglected. For the Newton procedure with $I$ iterations the evaluation of $\mathbb{S}^{\prime}\left(y_{i}[n]\right)=\mathbb{N}^{\prime}\left(y_{i}[n]\right)$ adds. If $\mathbb{N}$ is a Volterra system of order $P, \mathbb{N}^{\prime}$ is of order $P-1$, meaning that $M_{P-1, N}$ multiplications have to be added. Further, if the signal is of length $L$, an $L \times L$ matrix has to be inverted. This costs $\mathcal{O}\left(L^{3}\right)$ without simplifications. Setting the off-diagonal elements to zero reduces the complexity drastically. Only an additional division has to be performed, yielding therefore

$$
M_{N w t}=I\left(M_{P, N}+M_{P-1, N}\right)
$$


multiplications plus one division, a complexity that grows again linearly with the number of iterations $I$.

\section{Simulation Results}

In this section the successive approximation method based on the fixed-point formulation is compared with the here developed Newton method. The convergence speed and the robustness against model uncertainties (parameter errors and structure mismatch) are investigated via simulations. Both methods are then used to linearize a Volterra model, derived from measured input/output data from a power amplifier.

\section{A. Example 1: Convergence speed and model-parameter esti- mation errors}

The nonlinear system to linearize is a real-valued Volterra system of nonlinear order $P=3$,

$$
z[n]=\sum_{p=1}^{3} \sum_{n_{1}=0}^{2} \ldots \sum_{n_{p}=n_{p-1}}^{2} h_{p}\left[n_{1}, \ldots, n_{p}\right] \prod_{i=1}^{p} y\left[n-n_{i}\right] .
$$

Each kernel has a length of $N=3$ in each dimension (memory). The kernel weights are as follows:

$$
\begin{aligned}
h_{1}\left[n_{1}\right]= & \{0.5,0.01,-0.01\} \\
h_{2}\left[n_{1}, n_{2}\right]= & \{0.1,0.01,-0.02,0.005,-0.005,0.08\} \\
h_{3}\left[n_{1}, n_{2}, n_{3}\right]= & \{0.2,0.08,-0.05,0.01,-0.01, \\
& 0.005,0.01,0.01,-0.003,0.005\} .
\end{aligned}
$$

The indexing is according to (14). The excitation is a discrete multi-tone (DMT) signal with normalized bandwidth (with respect to sampling frequency) of $B=1 / 4$ and 16 tones, equally spaced.

1) Convergence speed: In Fig. 3 the convergence rate of the two methods is compared.

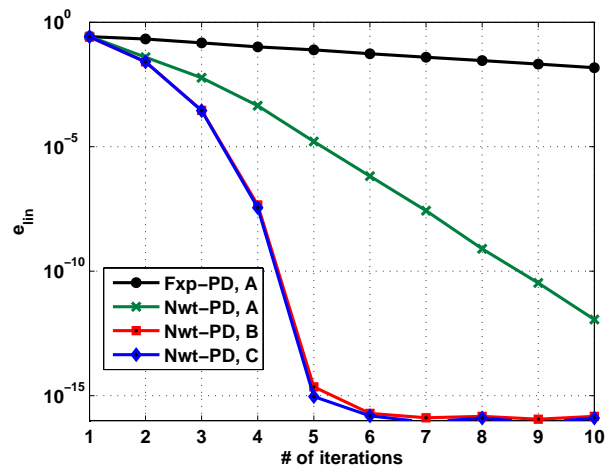

Fig. 3. Comparison of the convergence speed

The input signal is compared against the output of the linearized system, $z_{i}[n]=\mathbb{N}\left(\mathbb{P}_{i}(u[n])\right)$, $e_{\text {lin }}(i)=\left\|u[n]-z_{i}[n]\right\|_{2}$ after $i$ iterations, meaning that $\mathbb{L}=\mathbb{I}, \mathbb{I}$ denoting the identity operator. The Newton method outperforms the fixed-point method significantly with respect to convergence speed. Since the system is known, exact linearization is possible. Complexity reductions (only the main diagonal elements in $\mathbb{S}^{\prime}$ are used) without feeding back the pre-distorted signal $y_{I}[n]$, reduce the convergence rate of the Newton method (Nwt-PD, A), feeding back the signal $y_{I}[n]$ after the pre-distortion as depicted in Fig. 2, boosts the convergence rate again to quadratic (Nwt-PD, B). The performance is equivalent to the Newton method without modifications (Nwt-PD, C), operated in terms of operators. Feeding back the signal after the pre-distortion in the successive approximation method gives no improvements against the original method - the convergence rate cannot be larger than of linear order.

2) Model-parameter estimation errors: In real world applications the nonlinear system is seldom perfectly known. A system identification has to be performed which gives only a more or less accurate picture of the system. Two types of model uncertainties are investigated here: errors in the parameters, e.g., due to noisy measurements of the inputoutput signals, and model mismatch, e.g., assuming a lower order of the nonlinearity and/or memory length. In Fig. 4 the effects of parameter errors on the performance of the pre-distortion using the Newton method in its simplest form (Nwt-PD, B in Fig. 3) is reported. The parameter error $\varepsilon_{I}$ is the relative distance of the true system parameters, combined in the vector $\mathbf{h}$ and the model parameters, combined in $\hat{\mathbf{h}}$, $\varepsilon_{I}=20 \log \left(\frac{\|\mathbf{h}-\hat{\mathbf{h}}\|_{2}}{\|\mathbf{h}\|_{2}}\right) \mathrm{dB}$.

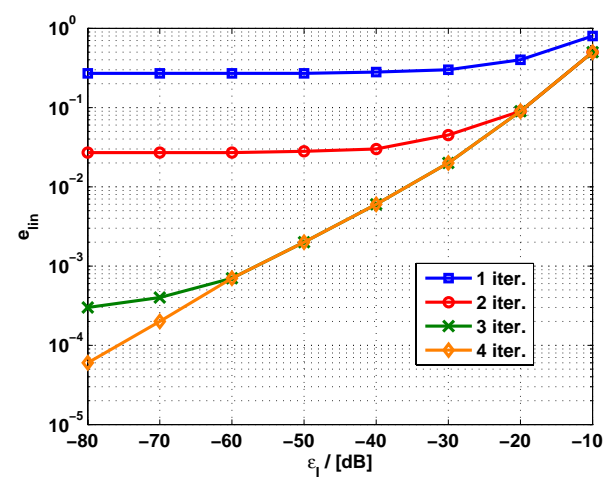

Fig. 4. Influence parameter-estimation errors on the linearization

With a reasonable system identification (e.g. $\varepsilon_{I}=-40 \mathrm{~dB}$ ), only three iterations are necessary to achieve the limit. With better identification accuracy, more iterations are to be performed in order to achieve the limit. The fixed-point method needs significantly more iterations to achieve the same performance and shows more sensitivity to modeling errors.

\section{B. Example 2: Model-structure mismatch}

If the model-structure does not match the system exactly, perfect linearization of the system cannot be achieved. In order to compare the two iterative linearization methods, a Wiener system, consisting of an FIR system of length three in front of a memoryless nonlinearity is modelled with a Volterra series. The linear system has the weights $h[0]=0.5, h[1]=$ 
$0.1, h[2]=0.02$. The memoryless nonlinearity following the linear system is a Taylor series of order 11

$$
z[n]=f(x[n])=\sum_{p=1}^{11} \alpha x[n]^{p}, \alpha=0.38,
$$

$x[n]$ denoting the output of the linear filter, $z[n]$ denoting the output of the whole system.

The Volterra model does not match the system exactly, i.e., the model order is lower than the system order - the Volterra series is simply truncated after $P=2, \ldots, 7$ elements. Fig. 5 shows that the achievable linearization error $e_{\text {lin }}=\left\|u[n]-z_{i}[n]\right\|_{2}$ of the Newton procedure decreases with increasing order of the Volterra model. If the order of the system is severely underestimated, a few iterations suffice to reach the achievable minimal error. The sensitivity of the fixed-point approach is higher to such modeling errors - the minimum achievable linearization error using a Volterra model of order greater than five is $5 \cdot 10^{-2}$ whereas using the Newton approach the achievable minimal linearization error is less than $5 \cdot 10^{-4}$, two orders of magnitude smaller, achievable with only three iterations, whereas the fixed-point method needs seven iterations.

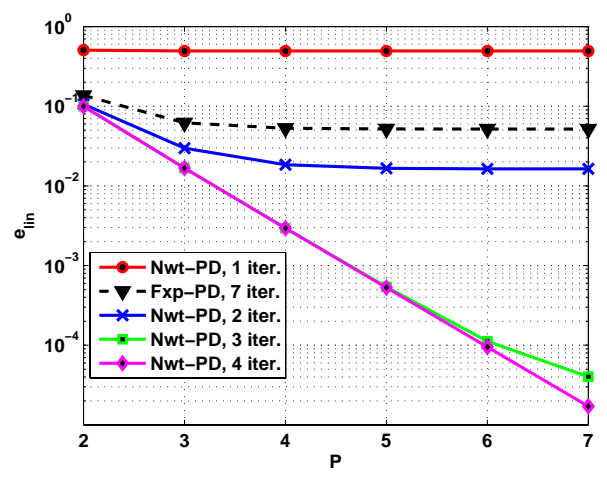

Fig. 5. Influence of model-structure mismatch

\section{Linearizing a real power amplifier}

Measured input/output data from a standard power amplifier (Minicircuits, Type: ZLH-42W) is now used to derive a Volterra model. After the modeling, the two presented methods are used to linearize the derived Volterra model.

The chosen test-signal is a multi-tone signal with a total bandwidth of $2 \mathrm{MHz}$, whereby the individual tones are separated by $20 \mathrm{kHz}$. The phases are uniformly distributed in the interval $[-\pi, \pi)$. A standard spectrum analyzer (Agilent PSA) is used to sample the signal at the output of the excited power amplifier. The sampling-rate $f_{s}$ used is $10.24 \mathrm{MHz}$. The Volterra model is of fifth order, whereby only the uneven terms are used. The memory lengths of the kernels are three for all uneven terms. In order to apply the Newton method, the model has to be analytic. The model used here is

$$
\begin{aligned}
z[n]= & \sum_{p} \sum_{\mathbf{n}_{1}} \sum_{\mathbf{n}_{2} \neq \mathbf{n}_{1}} h_{p}\left[\mathbf{n}_{1}, \mathbf{n}_{2}\right] \\
& \times \prod_{i=1}^{\lceil p / 2\rceil} y\left[n-\mathbf{n}_{1}[i]\right] \prod_{j=1}^{\lfloor p / 2\rfloor} y^{*}\left[n-\mathbf{n}_{2}[j]\right]
\end{aligned}
$$

where the index vectors are $\mathbf{n}_{1}=\left[n_{1}, \ldots, n_{\lceil p / 2\rceil}\right], \mathbf{n}_{2}=$ $\left[n_{\lceil p / 2\rceil+1}, \ldots, n_{p}\right]$. The notation $\mathbf{n}_{1} \neq \mathbf{n}_{2}$ means that the values of the indices have to be different. In this case $p=$ $1,3,5$ and $n_{i}=0,1,2$. This model requires in total 13 complex parameters. The achieved relative mean-squared error $M S E=20 \log \left(\frac{\|z[n]-d[n]\|_{2}}{\|d[n]\|_{2}}\right)$ in the modeling step $(d[n]$ is the output signal of the system, $z[n]$ is the output signal of the Volterra model), is $-26 \mathrm{~dB}$.

The Fig. 6 shows the AM/AM conversion (magnitude of the output signal vs. the magnitude of the input signal) with and without pre-distortion based on the successive approximation method, Fig. 7 shows the results achieved with the Newton method. The targeted linear gain is seven. With both methods four iterations are performed. It can be seen clearly that the Newton method corrects the dispersion (memory effects) better than the successive approximation method.



Fig. 6. AM/AM distortion with and without pre-distortion (PD)

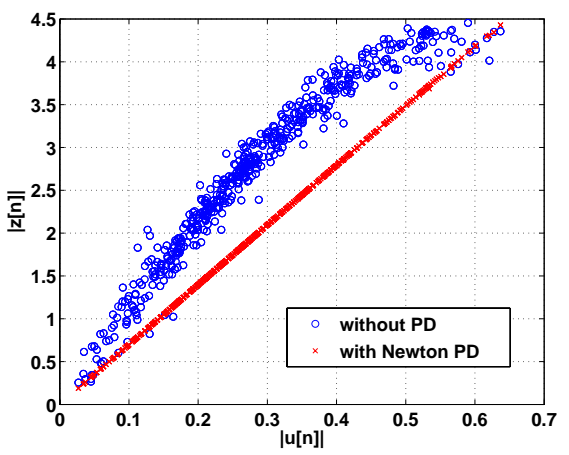

Fig. 7. AM/AM distortion with and without pre-distortion (PD)

The correction of the AM/PM conversion (difference of the input phase and the output phase, $\Delta \Phi=\arg (u[n])-\arg (z[n])$ vs. the input amplitude $|u[n]|)$ can be seen from Fig. 8 for 
the successive approximation method and from Fig. 9 for the Newton method. Also here the Newton method corrects the dispersion better than the successive approximation method.

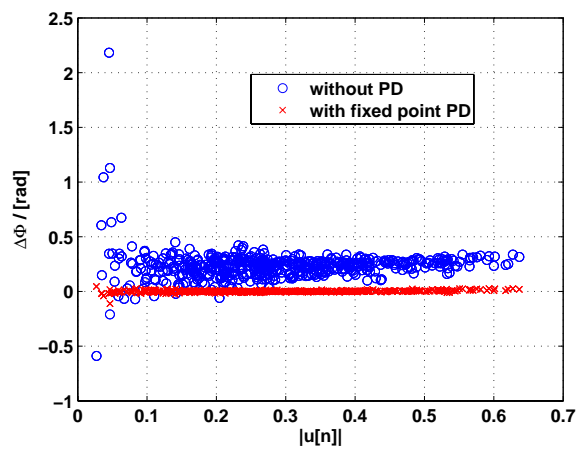

Fig. 8. AM/PM distortion with and without pre-distortion based on the successive approximation method



Fig. 9. AM/PM distortion with and without pre-distortion based on the Newton method

The spectra of the output signal of the Volterra model with and without pre-distortion can be seen in Fig. 10 and Fig. 11. The spectral regrowth is compensated nearly perfectly using the Newton method. The successive approximation method is not able to compensate the nonlinearity - more than four iterations would be needed which increases the complexity. Using the Newton method the third order components are up to $25 \mathrm{~dB}$ lower, the fifth order components up to $10 \mathrm{~dB}$ lower than by using the successive approximation method. The relative mean-squared error between the targeted output signal, $d_{l}[n]=7 \cdot u[n]$ and the achieved signal after four iterations $z_{4}[n], M S E=20 \log \left(\frac{\left\|d_{l}[n]-z_{4}[n]\right\|_{2}}{\left\|d_{l}[n]\right\|_{2}}\right)$, is for the Newton method $M S E_{N w t}=-48 \mathrm{~dB}$, for the successive approximation method $M S E_{F x p}=-31 \mathrm{~dB}$, a loss of $17 \mathrm{~dB}$.

\section{CONCLUSIONS}

An iterative pre-distortion algorithm based on the Newton algorithm was developed and compared with a successive approximation method based on an fixed-point equation, investigated in [5]. The complexity of the Newton method is higher than the complexity of the successive approximation method, but still grows linearly with the number of iterations. The

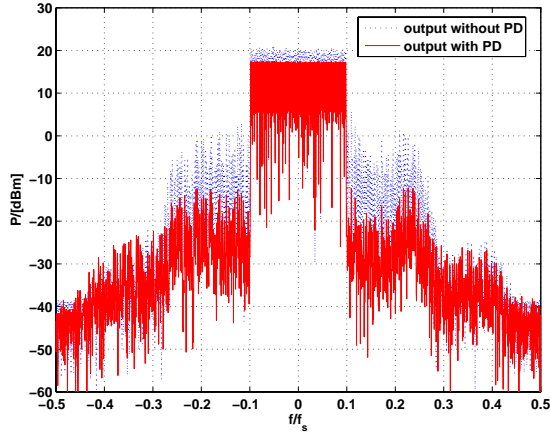

Fig. 10. Power spectra with and without fixed-point pre-distortion (PD) four iterations are performed

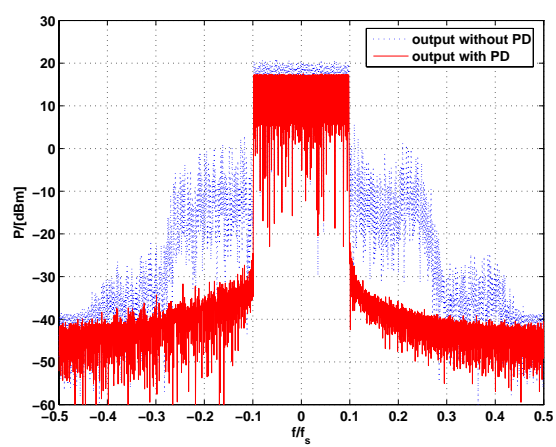

Fig. 11. Power spectra with and without Newton pre-distortion (PD) - four iterations are performed

convergence rate of the Newton method is quadratic, whereas the convergence of the successive approximation method is only linear, i.e., with the Newton method only few iterations are necessary to achieve a certain degree of linearity. Examples showed that the Newton method is more robust against modeling errors. Further, a Volterra model from a measured power amplifier is linearized with both methods. With only few iterations the spectral regrowth can be reduced nearly perfectly with the Newton method, whereas with the same number of iterations the successive approximation method yields a much higher spectral regrowth.

\section{REFERENCES}

[1] K. J. Muhonen, M. Kavehrad, and R. Krishnamoorthy, "Look-up table techniques for adaptive digital predistortion: A development and comparison," IEEE Trans. Veh. Technol., vol. 49, no. 5, pp. 1995-2002, Sep. 2000.

[2] J. Kim and K. Konstantinou, "Digital predistortion of wideband signals based on power amplifier model with memory," Electron. Lett., vol. 37, no. 23, pp. 1417-1418, Nov. 2001.

[3] E. Aschbacher and M. Rupp, "Identification on a nonlinear poweramplifier L-N-L structure for pre-distortion purposes," in Proc. IEEE International Symposium on Personal, Indoor and Mobile Radio Communications (PIMRC'03), Sep. 2003, pp. 2102-2106.

[4] M. Schetzen, The Volterra and Wiener Theories of Nonlinear Systems, John Wiley, 1980.

[5] R. D. Nowak and B. D. Van Veen, "Volterra filter equalization: A fixed point approach," IEEE Trans. Signal Processing, vol. 45, no. 2, pp. 377387, Feb. 1997.

[6] D. G. Luenberger, Optimization by Vector Space Methods, J. Wiley, 1968. 